\title{
Online Courses on COVID 19 Outbreak for Health Workers by World Health Organization- A Review
}

\author{
Dr. Sandeep Kale ${ }^{1 *}$, Dr. Govind Khati ${ }^{2}$, Dr. Pornima Kale ${ }^{3}$, Dr. Santosh Girbide ${ }^{4}$, Dr. Milind Kamble ${ }^{1}$ \\ ${ }^{1}$ Assistant Professor, Department of Rognidan Avum VikiritiVigyan, R. A. Podar Medical (Ayu) College, Worli Mumbai, India \\ ${ }^{2}$ Professor of Sanskrut Samhita Department \& Dean of R. A. Podar Medical (Ayu) College, Worli Mumbai, India \\ ${ }^{3}$ Ayurved and Yoga Consultatnt, Livelong Ayurved Clinic, Mumbai, India \\ ${ }^{4}$ Professor, Department of Rognidan Avum VikiritiVigyan, R. A. Podar Medical (Ayu) College, Worli Mumbai, India
}

\begin{abstract}
DOI: $10.36348 /$ sijtcm.2021.v04i03.001
| Received: 06.03.2021 | Accepted: 23.03.2021 | Published: 28.03.2021
\end{abstract}

*Corresponding author: Dr. Sandeep Kale

\section{Abstract}

The World Health Organization (WHO) is a agency which works for international public health. It is headquartered in Geneva, Switzerland, with six semi-autonomous regional offices and 150 field offices worldwide. The WHO has prio role in several public health achievements, most notably the eradication of smallpox, the near-eradication of polio, and the development of an Ebola vaccine. The WHO generated an Incident Management Support Team on 1 January 2020 , one day after Chinese health authorities identified the organization of a cluster of pneumonia cases of unknown etiology. Many countries are affected because of COVID-19 breakthrough and fighting with available resources. WHO has aligned branch OpenWHO which is interactive, web-based, knowledge-transfer platform offering online courses to improve the response to health emergencies. OpenWHO has created online courses to educate healthcares. So they more than thirty courses to learn and understand COVID 19 all aspects. These courses are online and we have to invest some time to learn via many videos and presentations. We can complete courses from minimum 1 hour to 10 hours depend on which course we choose. They provides us certificate after completion of $85 \%$ course.

Keywords: Online courses, OpenWHO, WHO, COVID 19, Journalism, Health workers.

Copyright (C) 2021 The Author(s): This is an open-access article distributed under the terms of the Creative Commons Attribution 4.0 International License (CC BY-NC 4.0) which permits unrestricted use, distribution, and reproduction in any medium for non-commercial use provided the original author and source are credited.

\section{INTRODUCTION}

World Health Organization i.e. WHO has aligned branch OpenWHO which is interactive, webbased, knowledge-transfer platform offering online courses to improve the response to health emergencies. OpenWHO aims the Organization and its key partners to give life-saving knowledge to abundant numbers of frontline responders. This platform provides online courses to people make ready to work in epidemics, pandemics and health emergencies or already doing so $[1]$.

This platform conveys WHO's and partners' expertise closer to where it is needed and sends information in user-friendly formats for frontline responders and decision-makers. The platform is handled by the Learning and Capacity Development unit in the WHO's Health Emergencies Programme. Interested individual take part in a worldwide, social learning network that is established on interactive, online courses and materials covering a variety of subjects. OpenWHO also carry out a forum for the fast sharing of public health expertise and in-depth discussion and feedback on key issues.

Many online training courses are prepared for health workers in which two courses are for journalism in COVID 19. Currently word is under COVID 19 outbreak. So, total 24 courses are outlined according to COVID 19 safety, risk, prevention, management, and vaccination. The current outbreak of novel corona virus disease has spread across borders through travelers, conveyances, which has affected point of entry so five courses are based upon COVID 19 at point of entry [2].

\section{Courses for health workers}

1. COVID-19 vaccination training for health workers All health workers related to implementation of COVID-19 vaccination need to acquire sufficient knowledge and skills in order to ensure safe and efficient COVID-19 vaccine administration.

2. Orientation to national deployment and vaccination planning for COVID-19 vaccines 
This module introduces the National Deployment and Vaccination Plan (NDVP) guidance document for COVID-19 vaccines.

3. Guidance on mask use in the context of COVID-19 This training is based on scientific evidence and WHO guidance relevant to the use of masks for reducing the spread of SARS-CoV-2 in different population.

4. COVID-19 and work: Staying healthy and safe at work during the COVID-19 pandemic.

This training is based on maintaining open, safe and healthy workplaces are of vital importance to protecting livelihoods, wellbeing and public health.

5. COVID-19 infodemic management: risk communication and community engagement challenges.

This course will focus on how countries, communities and individuals are directing public health and social measures by maintaining what's working while addressing challenges that still lie ahead for 2021.

6. Management and Facilitation of a Country COVID-19 Intra-Action Review (IAR).

This course gives a general introduction to the management and the facilitation of an IAR during the ongoing COVID-19 pandemic through presentations, quizzes and an assessment at the end of the course to test the knowledge acquired.

7. Introduction to emerging respiratory viruses, including novel coronavirus.

This course gives a overall introduction to COVID19 and emerging respiratory viruses and is intended for public health professionals, incident managers and personnel working for the United Nations, international organizations and NGOs.

8. Infection Prevention and Control (IPC) for novel coronavirus (COVID-19).

This training is focused on infection prevention and control for healthcare workers and public health professionals.

9. Health and safety briefing for respiratory diseases ePROTECT

This course gives a introduction of basic hygiene measures to protect against infection.

10. COVID-19: Operational Planning Guidelines and COVID-19 Partners Platform to support country preparedness and response.

These planning guidelines teaches priority steps and actions to be involved in countries' preparedness and response plans across the major areas of public health preparedness and response.

11. Clinical management of patients with COVID-19 General considerations.

This course gives crucial knowledge necessary to available safe, effective quality patient care. Presentations address all area of clinical management, including facility preparation and surge planning; health worker infection prevention and control; interfacility transfer; clinical management of mild, moderate, and severely ill patients with COVID-19; special considerations for geriatric, pregnant, and pediatric patients with COVID-19; rehabilitation; and ethics and palliative care.

12. Clinical management of patients with COVID-19 Rehabilitation of patients with COVID-19.

This course address rehabilitation needs of patients recovering from COVID-19, such as cognitive impairment, physical weakness, respiratory impairment, swallow impairment, communication impairment and challenges in completing Activities of Daily Living (ADLs).

13. Neglected tropical diseases in the context of the COVID-19 pandemic: impact and guidance.

This course is made for decision-makers and programme managers who want to learn more about neglected tropical diseases (NTDs).

14. Severe Acute Respiratory Infection (SARI) Treatment Facility Design.

This includes Severe Acute Respiratory Infection (SARI) outbreak preparedness, readiness and response.

15. WHO COVID-19 Mass Gatherings Risk Assessment Training.

The aim of this course contains study of risks association with transmission of this infection.

16. Occupational health and safety for health workers in the context of COVID-19.

All health workers aware of knowledge and skills to protect themselves and others from the occupational they encounter, so that they can work safely and effectively.

17. Long-term care facilities in the context of COVID19.

The COVID-19 pandemic shows effect on older people disproportionately, especially those living in long-term care facilities (LTCF) with significant impact on mortality and morbidity. So, concerted action is needed to mitigate the impact of COVID19 by enhancing infection prevention and control (IPC) measures within LTCF.

18. Introduction to Go.Data - Field data collection, chains of transmission and contact follow-up This training gives an orientation to the purpose, benefits and utilization of Go.Data. Which is a narrated walkthrough of the key features of the Go.Data web-based platform and mobile application.

19. COVID-19: How to put on and remove personal protective equipment

This is learning for healthcare workers busy in patient care activities in a healthcare setting. It narrates the type of personal protective equipment or PPE needed to correctly protect oneself.

20. Standard precautions: Hand hygiene

Cleaning hands at the right times and in the right way is a major factor for prevention of infection. The WHO Guidelines on hand hygiene in health care support hand hygiene promotion and improvement in health care facilities worldwide. 
21. Standard precautions: Waste management

According to WHO, about $85 \%$ of the total amount of waste created by health care activities is general, non-hazardous waste. The remaining $15 \%$ is considered hazardous material that could be infectious, toxic or radioactive. Hazardous waste that is not throughout properly which presents a risk to hospital patients, health care personnel and the general public.

22. Clinical Care Severe Acute Respiratory Infection. This course teaches on clinical management of patients with a severe acute respiratory infection.

\section{Other training courses for journalists:}

The course is free and available to journalists who are interested for new knowledge and resources to increase our coverage and understanding of the pandemic. The course is based on following topics like, where did COVID-19 come from? Covering COVID-19 right now, the hope for treatments and vaccines, and webinar on covering the COVID-19 vaccine and what journalists need to know? This webinar was presented by Knight Center for Journalism in the Americas in partnership with UNESCO \& the World Health Organization, with funding from the European Union. The course has introduced by following names.

1. Journalism in a pandemic: Covering COVID-19 now and in the future -- A self-directed course for journalists.

2. Webinar: Covering the COVID-19 vaccine: What journalists need to know

\section{Five online courses on COVID-19 at Points of Entry}

Vessels that transport goods and carry no passengers and fishing vessels confront particular challenges in carrying out their functions and maintaining their health in the time of the COVID-19 pandemic. In some cases, they take ship on extended tour without calling at any port for long periods. An outbreak on board a ship is a concern for the safety and well-being of the crew and may affect the crew's ability to safely navigate and operate the ship.

\section{Controlling the Spread of COVID-19 at Ground Crossings}

At the end of this course, we should be competent to reduce the spread of COVID-19 resulting from travel, transportation, and trade on and around ground crossings by Identifying priority ground crossings and communities; and Scaling up preparedness and control measures at these locations.

2. Operational Considerations for Managing COVID-19 Cases and Outbreaks in Aviation.

At the end of this course, we should be competent to draft an airport public health contingency plan for managing COVID-19 cases and outbreaks in aviation and manage an outbreak of COVID-19 disease in aviation.
3. Management of ill travelers at points of entry in the context of the COVID-19 outbreak.

The current outbreak of novel corona virus disease has spread across borders through travelers, conveyances, which has prompted demands for the detection and management of suspected cases at points of entry (POE), including ports, airports and ground crossings, on board conveyances.

At the end of this course, we should be competent to outline the core capacities to be in place for managing ill travelers suspected to have COVID19 at points of entry and on conveyances of all types and put in place mechanisms that facilitate the management of ill travelers suspected to have COVID-19 at points of entry and on conveyances of all types.

4. Operational Considerations for Managing COVID-19 Cases and Outbreaks on Board Ships.

At the end of this course, we should be competent to draft an outbreak management plan for managing COVID-19 cases and outbreaks onboard ships, manage an outbreak of COVID-19 disease on board a ship and perform follow-up epidemiological and environmental investigation.

5. Promoting Public Health Measures in Response To COVID-19 on Cargo Ships and Fishing Vessels

At the end of this course, we should be able to describe the public health measures that may protect seafarers throughout their journey to the vessel, on the vessel, and on the way home and prevent and manage an outbreak of COVID-19 disease on board a cargo ship or fishing vessel

In this course target audience are National IHR Focal Points (NFPs), authorities responsible for implementing the IHR at ground crossings, Representatives of government and non-government organizations and their partners at ground crossings, Public health professionals involved in disease surveillance, communication, emergency preparedness and response, animal health and environmental health at ground crossings and in nearby communities, Airport health authorities and local, provincial, and national health surveillance and response systems, Civil aviation authorities, airport operators, aircraft operators, airports, and airlines, conveyance operators, Ship owners, Seafarers, Unions and associations, Competent authorities for health and transport.

\section{About registration and access of these courses \\ Anyone access OpenWHO via the url; openwho.org [1] or download the OpenWHO App in Google \\ play: https://play.google.com/store/apps/details?id=de.x ikolo.openwho\&hl=en_GB. Those who are registers with their email address can access free OpenWHO courses. You can take as much time as you need to complete a course, no time limit. There are three levels}


of courses; basic, intermediate and advanced, under four channels offered by OpenWHO. For channels are Outbreak, Go Social, Ready for Response and Preparing for pandemics.

1. Outbreak: introduces knowledge and tools on specific diseases.

2. Go social: focuses on cross-cutting interventions such as risk communication.

3. Ready for response: talks about humanitarian response and how best to engage in emergency situations.

4. Preparing for pandemics: brings together courses on various aspects of influenza events.

OpenWHO also produces special knowledge resources packages developed from existing content during emergencies. OpenWHO has a mobile app, for both iOS and Android devices. OpenWHO can also be viewed via the internet browser on your mobile device. Some content, such as course presentations and videos, can be downloaded and viewed offline on the OpenWHO app. You can download content from the mobile app for offline use, simply navigate to the video or slide presentation. Scroll down to the bottom of the page. Under the heading 'Learning material', you could see which learning materials are available. By clicking on the three dots on the bottom right hand side of the screen, you can download these materials.

Many courses are made-up of a combination of downloadable video lectures and presentation slides, as well as self-assessments to test your knowledge. Discussion forums are also useful for users to share insight with experts in the field. A knowledge resource package for the use of health emergency response usually made up of presentations in the format of PDFs, shared with audio recordings in the language spoken by the local communities. OpenWHO has courses in multiple languages, including many of the UN official languages and the local languages of communities affected by particular outbreaks like Arabic, Chinese, English, French, Portuguese, Russian, and Spanish. Here we check the language of a particular course, navigate to the 'Course details' page. On the bottom right hand side of the course header image, the course language is displayed following the title "Language".

OpenWHO allocates certificates of completion for certain courses. This information is available on the course landing page, under 'Course details'. Click on 'Course details', check the information under the subtitle 'Course Requirements' or scroll to the bottom of the page, and read the information under the subtitle 'Certificate'. OpenWHO gives two types of certificates: records of achievement and confirmations of participation. You can find out which certificate, if any, is offered by our course, navigate to the 'Course details' tab on the course navigation toolbar. Some courses on OpenWHO allocate a record of achievement rather than a confirmation of participation. If we scored at least $80 \%$ on all course assessments then we are eligible for a record of achievement. Other courses only offer a confirmation of participation, which needs course participants to complete at least $80 \%$ of the course material. [2]

Name of the institution(s) that granted the approval: Not Required

Acknowledgements: World Health Organization and OpenWHO

\section{Conflicts of interest: None}

Sources of financial support that require acknowledgment: No Financial support for this study.

\section{REFERENCES}

1. https://openwho.org, cited on 10 March 2021

2. https://www.who.int/emergencies/diseases/novelcoronavirus-2019/training/online-training, cited on 11 march 2021 . 